\title{
Nonvitamin K oral anticoagulants in cardiac surgery: What cardiothoracic surgeons need to know 2.0
}

Stephen D. Waterford, MD, MS, ${ }^{a}$ and Niv Ad, $\mathrm{MD}^{\mathrm{b}}$

The use of nonvitamin K oral anticoagulants (NOACs) for various clinical indications is increasing in medical practice. ${ }^{1-3}$ However, there is scarcity in studies specifically designed to address the safety and efficacy of NOACs in patients undergoing cardiac surgery. We sought to address the current evidence gaps through a set of clinical questions:

- When should NOACs be discontinued before cardiac surgery to minimize the risk of perioperative bleeding?

- When anticoagulation treatment is indicated following cardiac surgery, is it safe to use NOACs instead of the traditional warfarin?

- How should the gaps in evidence be addressed to better equip cardiac surgeons' decision making regarding choice of anticoagulation treatment?

\section{BACKGROUND}

In 2014, Kaneko and colleagues ${ }^{4}$ addressed the use in cardiac surgery of a new class of oral anticoagulants, now called NOACs and also known as direct oral anticoagulants. At this time, NOACs were newly approved, and Kaneko and colleagues $^{4}$ discussed the properties of each of the NOACs, and reviewed the trials used to support their Food and Drug Administration (FDA) approval. They also discussed issues pertinent to cardiac surgery, including the perioperative management of NOACs. Since that time, reversal agents for NOACs have been approved and the use of NOACs has increased, although evidence addressing NOAC use in cardiac surgery patients is lacking. Guidelines have been published on preoperative discontinuation of NOACs before cardiac surgery, new series have appeared in the literature concerning NOACs in cardiac surgery, and new questions about NOACs have emerged for cardiac surgeons to answer. We provide an update for cardiac surgeons on the latest data on NOAC use in cardiac surgery, and suggest further studies to establish safe practices for NOAC use.

NOACs were first used in Europe. Dabigatran and rivaroxaban were approved in the European Union in 2008,

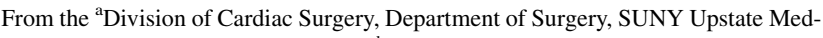
ical University, Syracuse, NY; and ${ }^{b}$ Division of Cardiac Surgery, Department of Surgery, Adventist HealthCare, Silver Spring, Md.

Received for publication Feb 2, 2021; revisions received Feb 25, 2021; accepted for publication March 8, 2021; available ahead of print March 19, 2021.

Address for reprints: Stephen D. Waterford, MD, MS, Division of Cardiac Surgery, Department of Surgery, 739 Irving Ave, Suite 640, Syracuse, NY 13210 (E-mail: waterfos@upstate.edu).

J Thorac Cardiovasc Surg 2022;163:2145-52

$0022-5223 / \$ 36.00$

Copyright (c) 2021 by The American Association for Thoracic Surgery

https://doi.org/10.1016/j.jtcvs.2021.03.059
}

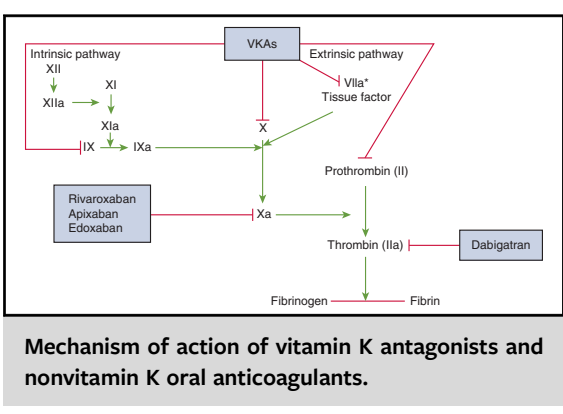

CENTRAL MESSAGE

Use of NOACs has increased in cardiac surgery, and further trials are needed to examine their safety and efficacy.

PERSPECTIVE
Use of NOACs has increased in cardiac surgery,
both as a preoperative medication, and postoper-
ative anticoagulation therapy. The precise risks
and benefits of NOAC use around the time of car-
diac surgery remain unknown, and further trials
are needed to examine their safety and efficacy.
See Commentary on page $\mathbf{2 1 5 3 .}$

followed by apixaban in 2011 and edoxaban in 2015 . Australia similarly approved dabigatran, rivaroxaban, and apixaban between 2008 and 2011. The FDA approved dabigatran in 2010, followed by rivaroxaban in 2011, apixaban in 2012, and edoxaban in 2015. Today NOACs are approved for deep vein thrombosis, pulmonary embolism, and nonvalvular atrial fibrillation. Since their initial approval, NOAC use has become very popular and has surpassed warfarin use for anticoagulation in a variety of indications. A nationwide review of NOAC use in the United Kingdom has shown that NOAC use increased 17-fold from 2012 to 2015 , whereas new warfarin prescriptions declined by $31 \%$ from 2009 to 2015 . In 2015, NOAC prescriptions accounted for $56.5 \%$ of all oral anticoagulant prescriptions in the country. ${ }^{1}$ Similarly, a review of 50 million beneficiaries in the French health care system demonstrated that of 1.1 million patients with atrial fibrillation who started anticoagulation therapy in 2016, 66.3\% were started on an NOAC. ${ }^{2}$ Notably, $40 \%$ of NOAC users were prescribed a reduced dose, illustrating the variable dosing of these 


\section{Abbreviations and Acronyms \\ ACC = American College of Cardiology \\ AHA $=$ American Heart Association \\ BMI = body mass index \\ $\mathrm{CABG}=$ coronary artery bypass graft \\ EACTS $=$ European Association for Cardio- Thoracic Surgery \\ EHRA $=$ European Heart Rhythm Association \\ FDA $=$ Food and Drug Administration \\ HRS = Heart Rhythm Society \\ NOAC $=$ nonvitamin $\mathrm{K}$ oral anticoagulant \\ $\mathrm{PT}=$ prothrombin time \\ STS = Society of Thoracic Surgeons \\ TAVR $=$ transcatheter aortic valve replacement}

medications. In the global registry on long-term antithrombotic treatment of atrial fibrillation, $47.6 \%$ of those with atrial fibrillation were prescribed a NOAC, whereas $32.8 \%$ were prescribed warfarin during a period from 2011 to $2014 .^{3}$ Many of these articles are already a few years old, and NOAC use is likely even more prevalent today. Although there are no reports that comment specifically on the percentage of cardiac surgery patients on a NOAC preoperatively, the overall trend is to greater use. It is therefore clear that NOAC use is here to stay, and cardiac surgeons will need information to help them manage these agents safely.

The surge in NOACs use has many causes. The absence of routine laboratory monitoring is among the largest, and the reduction in food and medication interactions is another. In addition, American Heart Association (AHA), American College of Cardiology (ACC), and Heart Rhythm Society (HRS) guidelines state that NOACs are recommended over warfarin in NOAC-eligible patients with atrial fibrillation, except in those with moderate-to-severe mitral stenosis or a mechanical heart valve. This carries a level IA recommendation, and is part of the 2019 focused update of the 2014 AHA/ACC/HRS guidelines for management of patients with atrial fibrillation. ${ }^{5}$ Support for this recommendation is based on the findings that NOACs are superior to warfarin in preventing stroke and thromboembolism in atrial fibrillation, and show a reduction in intracranial bleeding compared with warfarin. Further, NOACs may be associated with reduced risk of adverse renal outcomes compared with warfarin in these patients, and specifically among older patients, dabigatran has been associated with a lower risk of osteoporotic fracture than warfarin. ${ }^{5} \mathrm{Simi}-$ larly, the American College of Chest Physicians 2016 guidelines recommend NOACs over warfarin for treatment of deep vein thromboses and pulmonary emboli in patients without cancer. ${ }^{6}$ Therefore, owing to suggestion of improved efficacy, and lower risk profile, NOAC use will continue to rise. Table 1 shows the strengths and weaknesses of NOACs compared with warfarin, highlighting many of these issues.

NOACs involve some considerations for renal function when being prescribed (Table 2). Further, it has been shown that hemodialysis can be used to reduce dabigatran concentration in the blood by $49 \%$ to $68 \%$ with a single dialysis session, ${ }^{7}$ but not the other NOACs. Dabigatran can be reversed with idarucizumab, and the other NOACs can be reversed with andexanet alfa (Table 2). Both of these agents are FDA approved for use in the United States.

There are also dosing concerns with NOACs in morbidly obese patients with a body mass index (BMI) over 35 . Although weight was not an exclusion in any of the NOAC trials in atrial fibrillation or venous thromboembolism, there are case reports of treatment failure with low serum levels of dabigatran with severe obesity (BMI $\geq 40) .{ }^{8}$ By contrast, patients with $\mathrm{BMI} \geq 30 \mathrm{had}$ a trend to better outcomes with apixaban, and subgroup analysis by weight for edoxaban in acute venous thromboembolism shows no difference in safety or efficacy. ${ }^{8}$ Despite acknowledgement of these equivocal data, the European Heart Rhythm Association (EHRA) recommends that use of warfarin be considered in patients with BMI $\geq 40$ or weight $>120 \mathrm{~kg}$. If an NOAC is chosen in these patients, measurement of drug trough levels is advised, although there are no clinical data to correlate drug trough levels with outcomes. ${ }^{8}$ Similarly, the 2019 AHA/ACC/HRS guidelines recommend consideration of measurement of serum NOAC levels in patients with BMI $>35$ or weight $>120 \mathrm{~kg} .^{5}$

There are further medication-specific dosing concerns with each NOAC. Rivaroxaban capsules contain lactose and patients with lactose intolerance should not take rivaroxaban. ${ }^{9}$ In addition, rivaroxaban absorption decreases by $66 \%$ to $100 \%$ under fasting conditions, and it should be taken with a meal. ${ }^{10}$ The implications of this for patients with poor oral intake or ileus after cardiac surgery remain unclear, but it is possible that this issue may render apixaban a better choice postoperatively. Next, in a 2018 study of patients with atrial fibrillation, edoxaban was shown to have lower efficacy than warfarin in patients with creatinine clearance $>95 \mathrm{cc} / \mathrm{min}$, with edoxaban treatment associated with increased stroke and systemic embolism in this subgroup. ${ }^{11}$ As a result, FDA issued a black box warning for edoxaban for atrial fibrillation in patients with estimated creatinine clearance $>95 \mathrm{cc} / \mathrm{min}$. Finally, most factor Xa inhibitors are cleared predominantly by the liver (apixaban $75 \%$, rivaroxaban $65 \%$, and edoxaban $50 \%$ ) and dabigatran has $20 \%$ hepatic clearance, and the EHRA currently recommends that all NOACs not be used in Child-Pugh C cirrhosis. Rivaroxaban was also not recommended for use in Child-Pugh B cirrhosis. However, a more recent study has shown improved outcomes in cirrhotic patients with 
TABLE 1. Strengths and weaknesses of nonvitamin K oral anticoagulants (NOACs) compared with warfarin

\begin{tabular}{ll}
\hline \multicolumn{1}{c}{ Strengths of NOACs } & \multicolumn{1}{c}{ Weaknesses of NOACs } \\
\hline No routine laboratory monitoring tests & Difficult to assess activity, particularly before emergency surgery \\
Reduced food and medication interactions & Activity level dependent on renal function \\
Reduced overall bleeding and intracranial bleeding in atrial fibrillation & More limited availability of reversal agents \\
Better prevention of stroke and thromboembolism in atrial fibrillation & Currently contraindicated for use with mechanical valves \\
\hline
\end{tabular}

atrial fibrillation treated with NOACs compared with warfarin, and further studies are needed to clarify the role of NOACs in patients with cirrhosis. ${ }^{12}$

NOACs have a different mechanism of action than warfarin (Figure 1), and consequently their level of activity is more challenging to measure. The standard assays such as prothrombin time (PT)/international normalized ratio, activated partial thromboplastin time, and thrombin time are of limited utility in measuring NOAC activity. ${ }^{13}$ For example, the oral direct thrombin inhibitor dabigatran prolongs PT, but this effect varies with use of different reagents. The ecarin clotting time is a better method to assess dabigatran activity because ecarin clotting time has a closer correlation with plasma dabigatran levels, but is impractical owing to lack of widespread availability. Likewise, the 3 factor Xa inhibitors: rivaroxaban, apixaban, and edoxaban, have been found to prolong PT and activated partial thromboplastin time but not in a consistent manner that accurately reflects their level of activity. Methods using antifactor Xa activity assays have been reported for these 3 medications, but there is lack of consensus on their use. Therefore, whereas patients with NOACs enjoy the advantage of having no routine laboratory monitoring, it is difficult to ascertain the anticoagulant status of a patient at specific times, such as before emergency surgery, or after a period of holding the medication before elective surgery. This remains an issue for the cardiac surgeon in the perioperative management of NOACs because it can be difficult to know how long these medications should be stopped before cardiac surgery. Finally, direct measurement of NOAC plasma drug concentrations is impractical in clinical practice. Variable renal function further complicates matters, given that all NOACs are excreted to some extent by the kidneys.

Despite evidence for improved efficacy and safety in other areas, NOACs are currently contraindicated for mechanical valves. The landmark Randomized, Phase II Study to Evaluate the Safety and Pharmacokinetics of Oral Dabigatran Etexilate in Patients After Heart Valve Replacement (RE-ALIGN) study, published in 2013, randomized patients 2:1 to dabigatran versus warfarin after mechanical mitral valve replacement, mechanical aortic valve replacement, or both. ${ }^{14}$ Dabigatran dose $(150,220$, or $300 \mathrm{mg}$ twice daily) was selected based on kidney function, and was started a median of 6 days after surgery. The stroke rate was $5 \%$ in the dabigatran group versus $0 \%$ in the warfarin group, and major bleeding occurred in $4 \%$ in the dabigatran group versus $2 \%$ in the warfarin group. As a result, this trial was terminated prematurely. Of interest to cardiac surgeons, all major bleeding in the trial was pericardial. Although this outcome did not reach statistical significance, it represents some of the only available data on pericardial bleeding with dabigatran. Currently, the Prospective, Randomized, Active (Warfarin) Controlled, Parallel-Arm Clinical Trial to Determine if Participants With an On-X Aortic Valve Can Be Maintained Safely and Effectively on Apixaban (PROACT Xa) randomized controlled trial is underway to

TABLE 2. Nonvitamin K oral anticoagulant (NOAC) dosing and reversal

\begin{tabular}{|c|c|c|c|c|}
\hline NOAC & $\begin{array}{c}\text { Renal } \\
\text { clearance }(\%)\end{array}$ & Dosing based on renal function* & $\begin{array}{l}\text { Dialysis } \\
\text { to clear }\end{array}$ & Reversal agent \\
\hline Dabigatran & 80 & $\begin{array}{l}\mathrm{CrCl}>30,150 \mathrm{mg} \text { BID } \\
\mathrm{CrCl} 15-30,75 \mathrm{mg} \text { BID }\end{array}$ & Yes & Idarucizumab $\dagger, 5 \mathrm{~g} \mathrm{IV} \times 1$ \\
\hline Rivaroxaban & 66 & $\begin{array}{l}\mathrm{CrCl}>50,20 \mathrm{mg} \text { daily } \\
\mathrm{CrCl} 15-50,15 \mathrm{mg} \text { daily } \\
\mathrm{CrCl}<15 \text {, avoid }\end{array}$ & No & Andexanet alfa \\
\hline Apixaban & 25 & $\begin{array}{l}\text { Standard dose: } 5 \mathrm{mg} \text { BID } \\
\text { If } 2 \text { of following (age } \geq 80 \mathrm{y} \text {, weight } \leq 60 \mathrm{~kg} \text {, } \\
\quad \mathrm{Cr} \geq 1.5 \mathrm{mg} / \mathrm{dL} \text { ), then } 2.5 \mathrm{mg} \mathrm{BID} \S\end{array}$ & No & Andexanet alfa \\
\hline Edoxaban & 50 & $\begin{array}{l}\mathrm{CrCl}>50,60 \mathrm{mg} \text { daily } \\
\mathrm{CrCl} 15-50,30 \mathrm{mg} \text { daily }\end{array}$ & No & Andexanet alfa \\
\hline
\end{tabular}

$\mathrm{CrCl}$, Creatinine clearance; $B I D$, twice daily; $I V$, intravenous. *These reflect US Food and Drug Administration (FDA) prescribing information. Where a NOAC has multiple dosing regimens based on indication, those shown are for nonvalvular atrial fibrillation. $\dagger$ FDA approved in 2015. ‡FDA approved in 2018. §These recommendations represent the FDA prescribing information for apixaban. In practice, many providers reduce the dose of apixaban to $2.5 \mathrm{mg}$ BID if only renal dysfunction is present. 


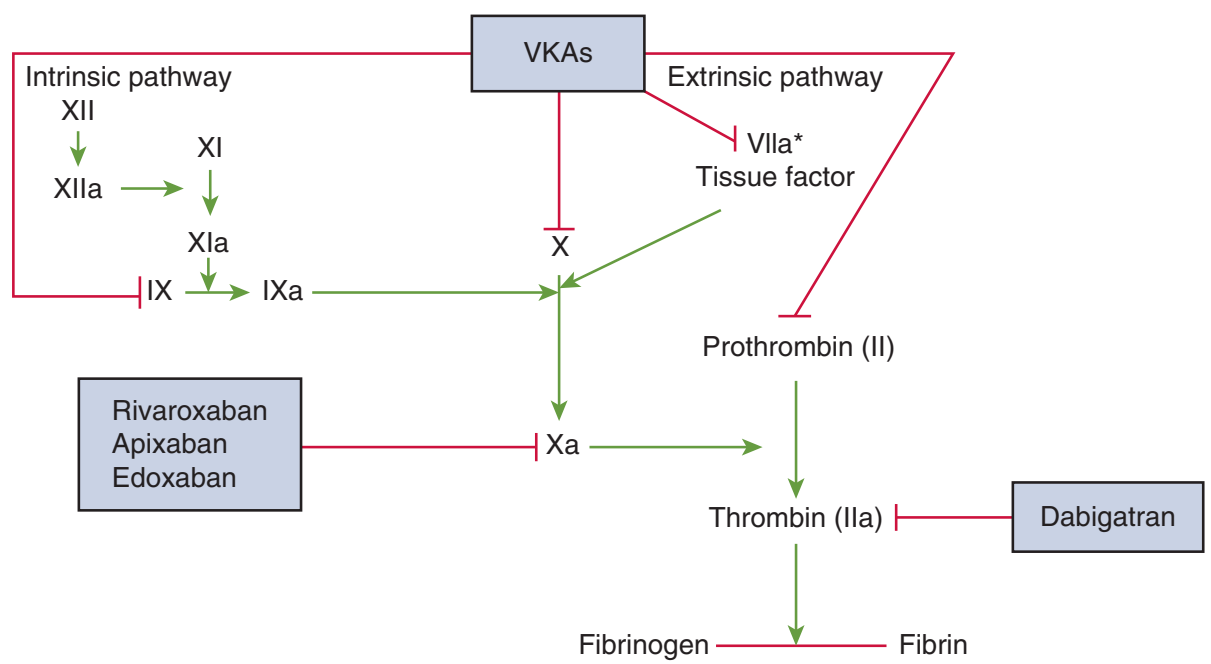

FIGURE 1. Mechanism of action of vitamin K antagonists (VKAs), the direct thrombin inhibitor dabigatran, and the factor Xa inhibitors rivaroxaban, apixaban, and edoxaban. Reprinted from Mekaj YH, Mekaj AY, Duci SB, Miftari EI. New oral anticoagulants: their advantages and disadvantages compared with vitamin K antagonists in the prevention and treatment of patients with thromboembolic events. Ther Clin Risk Manag. 2015;11:967-77. Noncommercial uses of the work are permitted without further permission from Dove Medical Press Limited.

compare apixaban to warfarin for the On-X aortic mechanical valve (On-X Life Technologies, Inc, Austin, Tex) ${ }^{15}{ }^{15}$ sults from this trial are eagerly awaited.

\section{WHEN SHOULD NOACS BE DISCONTINUED BEFORE CARDIAC SURGERY TO MINIMIZE THE RISK OF BLEEDING?}

There are no clear recommendations regarding the timing for NOAC discontinuation before cardiac surgery, and whether bridging with intravenous or subcutaneous heparin is required. The European Association for Cardio-Thoracic Surgery (EACTS) published guidelines for discontinuation of NOACs before elective cardiac surgery ${ }^{16}$ (Table 3). To summarize, these 2017 guidelines recommend holding oral factor Xa inhibitors (ie, rivaroxaban, apixaban, and edoxaban) for at least 48 hours, with no other specific recommendations. In addition, despite the different renal clearance of Xa inhibitors (Table 2), there are no specific guidelines for patients with renal dysfunction. With dabigatran, EACTS guidelines recommend holding for at least 48 hours for normal renal function, more than 72 hours (ie, $>3$ days) for mildly diminished renal function, and at least 96 hours for markedly diminished renal function
(Table 3). Although these guidelines address renal function, they do not address whether the duration of cessation should depend on the clinical indication for the NOAC to begin with. Patients with prior pulmonary emboli (with or without an inferior vena cava filter) are another group whose preoperative NOAC management is unclear. Finally, whether the duration of cessation should depend on the $\mathrm{CHA}_{2} \mathrm{DS}_{2^{-}}$ VASc score (ie, congestive heart failure, hypertension, age 65-74 years or $\geq 75$ years, diabetes mellitus, stroke/ transient ischemic attack [double weight], vascular disease, and sex category [female]) is also unknown.

By contrast, the American Association for Thoracic Surgery and Society of Thoracic Surgeons (STS) have not developed guidelines on perioperative management of NOACs. The Transfusion and Hemostasis Subcommittee of the European Association of Cardiothoracic Anesthesiology published a consensus statement on preoperative management of NOACs before cardiac surgery. ${ }^{17}$ They suggest that NOACs can be safely discontinued 2 days before cardiac surgery, on the basis of this being 5 half-lives for most NOACs. They suggest that in patients with renal or hepatic impairment or at high risk of bleeding, a preoperative plasma level below $30 \mathrm{ng} / \mathrm{mL}$ be verified for dabigatran,

TABLE 3. Nonvitamin K oral anticoagulant discontinuation before elective cardiac surgery in the European Association for Cardio-Thoracic Surgery (EACTS) guidelines

\begin{tabular}{|c|c|c|c|}
\hline Medication & $\begin{array}{l}\text { Discontinuation with } \\
\text { normal renal function }\end{array}$ & CrCl 50-79 mL/min & $\mathrm{CrCl}<50 \mathrm{~mL} / \mathrm{min}$ \\
\hline Dabigatran & At least $48 \mathrm{~h}$ & More than $72 \mathrm{~h}$ & At least $96 \mathrm{~h}$ \\
\hline Xa inhibitors: rivaroxaban, apixaban, edoxaban & At least $48 \mathrm{~h}^{*}$ & At least $48 \mathrm{~h}$ & At least $48 \mathrm{~h}$ \\
\hline
\end{tabular}

$\mathrm{CrCl}$, Creatinine clearance. *EACTS guidelines simply state to discontinue Xa inhibitors at least 48 hours before surgery. No specific time point is specified, and no modification for reduced renal function is made, despite different renal clearance of the different Xa inhibitors. 
apixaban, and rivaroxaban. If plasma level monitoring is not available, they suggest withdrawal of the NOAC for 4 days in these patients. They further suggest that for patients on a factor Xa inhibitor, an anti-Xa level $<0.1 \mathrm{IU} / \mathrm{mL}$ should be verified before cardiac surgery. Finally, they suggest that bridging with heparin is not required during the preoperative period. These guidelines are based mostly on the pharmacokinetics of NOACs, rather than data demonstrating safety of this approach with respect to postoperative bleeding.

In the noncardiac surgery literature, the EHRA recommends that NOACs should never be bridged with either unfractionated or low-molecular-weight heparin ${ }^{18}$ because bridging has been associated in the European Dresden NOAC Registry with higher procedural bleeding rates without lower thromboembolic rates. ${ }^{19}$ The Dresden NOAC Registry is a network of hospitals in Dresden, Germany, which enrolled patients on NOACs who subsequently underwent procedures varying from skin biopsies to pacemaker procedures to abdominal and thoracic surgery. In this registry, heparin bridging led to a higher rate of major bleeding $(2.7 \%)$ compared with no bridging $(0.5 \%)$, although these were noncardiac surgical procedures. Nonetheless, this provides some evidence that prior NOAC use might not be complemented well with a heparin bridge before major surgery, although these guidelines were based on studies in patients undergoing cardiology procedures, and further understanding is required in patients undergoing cardiac surgery.

One study that reports directly on NOAC discontinuation before cardiac surgery is a retrospective analysis of 81 consecutive patients who were taking a NOAC before cardiac surgery. ${ }^{20}$ Operations were performed a median of 4 days after NOAC discontinuation, and the duration of NOAC withdrawal was found to correlate with chest tube drainage over the first 24 hours after surgery. In this study, chest tube output declined significantly when the duration of NOAC withdrawal increased, all the way from 0 days to 14 days. On this basis, the authors suggest that NOACs should be withdrawn for at least 10 days before cardiac surgery where possible. These data are limited by the fact that the interquartile range of NOAC cessation was 3 to 6 days, and therefore conclusions on potential bleeding with a 10-day period of cessation are not supported because only interquartile range rather than the absolute range of cessation was provided in this study. They also found that reduced renal function was predictive of blood product administration in this cohort, potentially owing to incomplete clearance of NOACs in patients with reduced renal function. Finally, they demonstrated a re-exploration for bleeding rate of $6.2 \%$ in patients on preoperative NOACs, versus $3.3 \%$ in patients on warfarin or on dual antiplatelet therapy preoperatively. Although this particular outcome was not analyzed statistically, it suggests the need for further data on the contribution of preoperative treatment with NOACs to postoperative bleeding. To summarize, it remains unclear how long NOACs should be discontinued preoperatively because currently there are no reproducible methods to measure residual NOAC effect after withdrawal, unlike warfarin withdrawal. It is probably safe to assume that discontinuation of NOACs for at least 3 to 5 days before heart surgery would be sufficient, but there is a significant gap in evidence regarding the timing for discontinuation and the recommended bridging practice, as well as specific adjustments needed for patients with renal insufficiency.

\section{WHEN ANTICOAGULATION TREATMENT IS INDICATED FOLLOWING CARDIAC SURGERY, SHOULD CLINICIANS USE NOACS INSTEAD OF WARFARIN?}

Currently, a significant number of cardiac surgery centers and surgeons are reluctant to routinely use NOACs when anticoagulation is indicated immediately following cardiac surgery, citing lack of evidence for safety and the lack of consistent treatment recommendations if bleeding occurs.

However, despite of the gaps in clinical evidence, the use of NOACs following cardiac surgery is on a steady rise. A recent review of a regional STS database from 2011 to 2018 showed that among patients discharged on anticoagulation after bioprosthetic aortic or mitral valve replacement or coronary artery bypass grafting (CABG), $23 \%$ were discharged on a NOAC. ${ }^{21}$ Over the study period, NOAC use when anticoagulation treatment was indicated increased from $10.3 \%$ to $35.4 \%$. There was no detectable effect on reoperation rates for bleeding, and NOAC use was associated with a 0.9-day decrease in length of stay. A recent survey of the EHRA examined 16 centers across Europe, of whom $25 \%$ never use NOACs for anticoagulation of postoperative atrial fibrillation. ${ }^{22}$ Of the $75 \%$ that do use NOACs after cardiac surgery, $25 \%$ wait more than 4 days after surgery to start a NOAC, whereas only $12.5 \%$ of centers in the study would wait more than 4 days after surgery to start warfarin. These data reflect concern over bleeding with NOAC use after cardiac surgery. The 2017 EACTS guidelines note that there is evidence supporting a greater benefit of NOACs over warfarin in nonvalvular postoperative atrial fibrillation, including those with a bioprosthetic valve, but stop short of recommending NOACs for all such patients. ${ }^{16}$

In the transfemoral transcatheter aortic valve replacement (TAVR) literature, a cohort study suggests that among patients with atrial fibrillation after TAVR, apixaban was associated with fewer complications compared with a vitamin $\mathrm{K}$ antagonist. ${ }^{23}$ These complications were a composite of mortality, stroke, life-threatening bleeding, acute kidney injury, coronary obstruction, major vascular complications, and valve dysfunction requiring reintervention. The superior results in the apixaban arm were driven by a 
reduced incidence of stroke, life-threatening bleeding, and major vascular complications. By contrast, a randomized trial of rivaroxaban after TAVR suggested increased risk. The Global Study Comparing a Rivaroxaban-Based Antithrombotic Strategy to an Antiplatelet-Based Strategy After Transcatheter Aortic Valve Replacement to Optimize Clinical Outcomes (GALILEO) randomized trial, published in 2019, compared prophylactic-dose rivaroxaban (10 mg daily) plus aspirin (for the first 3 months), to aspirin plus clopidogrel (for the first 3 months), after TAVR. ${ }^{24}$ Eight hundred twenty-six patients were randomized to the rivaroxaban group, and 818 were randomized to the antiplatelet group. The trial found that rivaroxaban was associated with a higher risk of thromboembolic complications and a higher risk of bleeding than a dual antiplatelet strategy. As a result, the trial was terminated prematurely by the data and safety monitoring board. These patients had a median age of 80 years and an STS risk score in the rivaroxaban group of $4.0 \%$, and therefore differ from typical postcardiac surgery patients.

There are a few nonrandomized retrospective studies on NOACs after cardiac surgery. In a study from Australia of 106 CABG patients who were discharged receiving anticoagulation therapy for postoperative atrial fibrillation, $73 \%$ were prescribed warfarin and $27 \%$ prescribed an NOAC. There were no bleeding complications in patients discharged with a prescription for an NOAC, although this was a small study. ${ }^{25}$ In another retrospective review of 246 patients placed on oral anticoagulation therapy after isolated CABG, $74 \%$ received warfarin and $26 \%$ received an NOAC. Overall, $27 \%$ of those placed on NOACs required invasive interventions for pericardial or pleural effusions during the index hospitalization or up to 3 months postoperatively, compared with $13 \%$ of those receiving warfarin $(P<.014) .{ }^{26}$ An additional finding was that patients taking NOACs were more likely to required delayed interventions compared with those taking warfarin. A third study comes from the University of North Dakota, and was a retrospective review of patients undergoing isolated CABG who received anticoagulation therapy with NOACs or warfarin. ${ }^{27}$ That study found that neither group had a major bleeding event during index hospitalization. No patients receiving NOAC treatment had a delayed bleeding event, but there were 2 delayed bleeding events in the warfarin group. Total cost of care was significantly lower in the NOAC group.

The single randomized trial on use of NOACs after cardiac surgery was published in 2020 as a pilot study conducted in the United States, and also comes from the University of North Dakota. ${ }^{28} \mathrm{~A}$ total of 56 patients were randomized to receive a NOAC or warfarin for postoperative atrial fibrillation after cardiac surgery, and outcomes included transfusions and thoracentesis for pleural effusion. At these small numbers, there was no difference detected between the 2 groups, and there were no re-explorations for bleeding or pericardial drainage procedures in either group. Next, a review of the STS database examined 26,522 patients discharged taking anticoagulation therapy, of whom $63.2 \%$ received warfarin and $36.8 \%$ received a NOAC. ${ }^{29}$ In that retrospective review of nonrandomized patients, there was no difference between the 2 groups in 30-day major bleeding complications, pericardial effusion/tamponade, or stroke/transient ischemic attack, and there was a reduction in length of stay in the NOAC group from 8.7 to 8.2 days. This review demonstrates the rise in use of NOACs amongst cardiac surgeons.

Finally, it is unclear whether it is safe to resume NOACs postoperatively in patients who are taking NOACs preoperatively. The 2017 EACTS guidelines recommend that if a patient is taking a vitamin $\mathrm{K}$ antagonist or NOAC before surgery, that it be resumed after surgery. There is only a comment made that one should restart NOACs cautiously after cardiac surgery, due to the more immediate onset of antithrombotic effects and the increased risk for bleeding. ${ }^{16}$ Further, should there be bleeding on a NOAC postoperatively, it is not clear as to the methods and timing of reversal or whether reversal is necessary. Andexanet alfa requires a continuous infusion after a bolus to maintain reversal of factor $\mathrm{Xa}$ inhibitors, and is associated with considerable expense. ${ }^{30}$ Moreover, andexanet alfa has been shown to bind tissue factor pathway inhibitor, which could lead to thrombosis through enhanced thrombin formation, and in fact the package insert recommends monitoring for signs of arterial and venous thromboembolic events. ${ }^{31}$ Further, safety of andexanet alfa has not been evaluated in patients who have received recombinant factor VIIa or whole blood products within 7 days before administration. ${ }^{31}$ Idarucizumab, the reversal agent for dabigatran, irreversibly binds dabigatran within minutes of administration, but can require a second dose owing to redistribution of unbound dabigatran from the extravascular space to the intravascular space. ${ }^{30}$ Further, in the Reversal Effects of Idarucizumab on Active Dabigatran (RE-VERSE AD) trial, thrombotic events occurred in 34 of 503 patients receiving idarucizumab at 90 days, and therefore the prothrombotic consequences of reversal are unclear. ${ }^{32}$

\section{HOW SHOULD THE GAPS IN EVIDENCE BE ADDRESSED TO BETTER EQUIP CARDIAC SURGEONS' DECISION MAKING REGARDING CHOICE OF ANTICOAGULATION TREATMENT?}

NOAC use is on the rise in the community for anticoagulation, and current prescribing levels for NOACs exceed that for warfarin, a major shift during the past decade. It is now common to see patients in clinic receiving an NOAC before elective cardiac surgery. There remain 3 unanswered questions. First, how long should each NOAC be held for before cardiac surgery to produce safe outcomes and minimize postoperative bleeding? Second, when new anticoagulation 
TABLE 4. Future Studies for nonvitamin K oral anticoagulants (NOACs) in cardiac surgery

\begin{tabular}{|c|c|c|}
\hline Issue & Study & End points \\
\hline $\begin{array}{l}\text { Use of NOACs after cardiac surgery for atrial } \\
\text { fibrillation and bioprosthetic valves }\end{array}$ & $\begin{array}{l}\text { RCT of NOAC vs warfarin for atrial } \\
\text { fibrillation or bioprosthetic valve after } \\
\text { cardiac surgery }\end{array}$ & $\begin{array}{l}\text { Major bleeding events (return to operating } \\
\text { room or pericardial drainage procedure, } \\
\text { blood transfusion, chest tube output) } \\
\text { Thromboembolic events (stroke, TIA, } \\
\text { systemic emboli) }\end{array}$ \\
\hline $\begin{array}{l}\text { Duration of cessation of NOACs before } \\
\text { cardiac surgery }\end{array}$ & $\begin{array}{l}\text { RCT of } 2 \text { different durations of cessation ( } 2 \mathrm{~d} \\
\text { vs } 5 \mathrm{~d} \text { ) before elective cardiac surgery }\end{array}$ & $\begin{array}{l}\text { Bleeding (return to operating room or } \\
\text { pericardial drainage procedure, blood } \\
\text { transfusion, chest tube output) }\end{array}$ \\
\hline $\begin{array}{l}\text { Reversal of NOACs before urgent and } \\
\text { emergent cardiac surgery }\end{array}$ & $\begin{array}{l}\text { RCT of no reversal agent vs new reversal } \\
\text { agents andexanet alfa for factor Xa } \\
\text { inhibitors and idarucizumab for dabigatran }\end{array}$ & $\begin{array}{l}\text { Major bleeding events (return to operating } \\
\text { room or pericardial drainage procedure, } \\
\text { blood transfusion, chest tube output) } \\
\text { Thromboembolic events (stroke, TIA, } \\
\text { systemic emboli) }\end{array}$ \\
\hline
\end{tabular}

RCT, Randomized controlled trial; TIA, transient ischemic attack.

therapy is chosen after cardiac surgery, are NOACs a safe choice compared with warfarin? Third, if a patient was on a NOAC before cardiac surgery, is it safe to resume postoperatively, and if so, at what time and dose?

The issue of preoperative cessation of NOACs remains unanswered. Whereas EACTS has guidelines about the timing of cessation of NOACs before cardiac surgery, the evidence base is poor, and the American Association for Thoracic Surgery and STS have not made recommendations. The single study on chest tube drainage suggests decreased bleeding when the duration of NOAC withdrawal before surgery is lengthened to at least 10 days,${ }^{20}$ but the safety of this from the standpoint of thromboembolic events is unknown, and these data await further validation. In addition, there are a few retrospective series that address postoperative use of NOACs after cardiac surgery, but none are large in size or provide definitive answers. It remains unknown whether the rapid onset of therapeutic levels is safe when starting NOACs after cardiac surgery. Any increased bleeding with NOACs could be due to immediate onset of action. By contrast warfarin could lead to lower rates of bleeding, and better control of bleeding if it occurred. As a result, use of NOACs after cardiac surgery is left to individual provider discretion, and even dosing is unclear, with many surgeons using half-dose NOACs, or other regimens, after cardiac surgery. Finally, for postoperative cardiac surgery patients on a NOAC who develop bleeding, it is difficult to measure the activity levels of NOACs to decide about the use of a reversal agent or dialysis.

In short, 2 randomized trials of NOAC use in cardiac surgery are needed, to address the large segment of cardiac surgery patients who are on a NOAC preoperatively, or who require anticoagulation after cardiac surgery (Table 4). We would propose a first study wherein patients who receive anticoagulation therapy following cardiac surgery are randomized to an NOAC or to warfarin. Inclusion criteria would be prescription of anticoagulation for postoperative atrial fibrillation, or prescription of anticoagulation for a bioprosthetic valve replacement. We recommend a trial with apixaban because twice daily dosing more closely mirrors its half-life of 12 hours, compared with once daily dosing of rivaroxban, which has a shorter half-life than apixaban. There would be 3 arms to the study: prescription of a half dose NOAC for a week followed by full dose thereafter, prescription of a full-dose NOAC from the start, and prescription of warfarin. The reason for inclusion of a half dose NOAC arm is to address the concern that NOACs have immediate onset of action, potentially leading to increased bleeding. Should increased bleeding be seen in the full dose NOAC arm, the next trial to be requested would be a half-dose arm. Including this arm in the original study would therefore make the study more complete. The primary end points for such studies would be structured around the definitions of major bleeding events and the appropriate secondary safety end points.

In a second study, patients taking preoperative NOACs would be randomized to length of withdrawal of the medication. Each NOAC would have a different duration of withdrawal. For the factor Xa inhibitors rivaroxban and apixaban, a 2-day withdrawal period would be compared with a 5-day withdrawal period. For dabigatran in patients with normal renal function, a 2-day withdrawal period would be compared with a 5-day withdrawal period. For dabigatran in patients with creatinine clearance $<50 \mathrm{cc} / \mathrm{min}$, a 4-day withdrawal period would be compared with a 7-day withdrawal period. End points would be constructed around chest tube drainage, blood transfusion, and major bleeding events. Finally, we also propose a third study: use of reversal agents for patients taking NOACs who need urgent or emergent cardiac surgery. This study would help to clarify the role of new reversal agents in the management of patients on NOACs. These 3 studies would collectively provide the evidence base necessary to safely use NOACs in cardiac surgery. They would address the 3 major areas of concern in their use: holding, reversal, and resumption. 


\section{Conflict of Interest Statement}

The authors reported no conflicts of interest.

The Journal policy requires editors and reviewers to disclose conflicts of interest and to decline handling or reviewing manuscripts for which they may have a conflict of interest. The editors and reviewers of this article have no conflicts of interest.

\section{References}

1. Loo SY, Dell'Aniello S, Huiart L, Renoux C. Trends in the prescription of novel oral anticoagulants in UK primary care. Br J Clin Pharmacol. 2017;83: 2096-106.

2. Maura G, Billionnet C, Drouin J, Weill A, Neumann A, Pariente A. Oral anticoagulation therapy use in patients with atrial fibrillation after the introduction of non-vitamin $\mathrm{K}$ antagonist oral anticoagulants: findings from the French healthcare databases, 2011-2016. BMJ Open. 2019;9:e026645.

3. Huisman MV, Rothman KJ, Paquette M, Teutsch C, Diener H-C, Dubner SJ, et al The changing landscape for stroke prevention in AF: findings from the GLORIAAF registry phase 2. J Am Coll Cardiol. 2017;69:777-85.

4. Kaneko T, Yammine M, Aranki SF. New oral anticoagulants-what the cardiac surgeon needs to know. J Thorac Cardiovasc Surg. 2014;148:1794-801.

5. January CT, Wann LS, Calkins H, Chen LY, Cigarroa JE, Cleveland JCJR, et al. 2019 AHA/ACC/HRS focused update of the 2014 AHA/ACC/HRS guideline for the management of patients with atrial fibrillation: a report of the American College of Cardiology/American Heart Association Task Force on clinical practice guidelines and the Heart Rhythm Society in collaboration with the Society of Thoracic Surgeons. Circulation. 2019;140:e125-51.

6. Kearon C, Akl EA, Ornelas J, Blaivas A, Jimenez D, Bounameaux H, et al. Antithrombotic therapy for VTE disease: CHEST guideline and expert panel report. Chest. 2016;149:315-52.

7. Jain N, Reilly RF. Clinical pharmacology of oral anticoagulants in patients with kidney disease. Clin J Am Soc Nephrol. 2019;14:278-87.

8. Steffel J, Verhamme P, Potpara TS, Albaladejo P, Antz M, Desteghe L, et al. The 2018 European Heart Rhythm Association practical guide on the use of nonvitamin $\mathrm{K}$ antagonist oral anticoagulants in patients with atrial fibrillation. Eur Heart J. 2018;39:1330-93.

9. Baglin T. Clinical use of new oral anticoagulant drugs: dabigatran and rivaroxaban. Br J Haematol. 2013;163:160-7.

10. Beyer-Westendorf J, Siegert G. Of men and meals. J Thromb Haemost. 2015; 13: 943-5.

11. Yu HT, Yang PS, Kim TH, Jang E, Kim D, Uhm JS, et al. Impact of renal function on outcomes with edoxaban in real-world patients with atrial fibrillation. Stroke. 2018;49:2421-9.

12. Gallagher C, Sanders P, Wong CX. Anticoagulation for atrial fibrillation in cirrhosis of the liver: are low-dose non-vitamin $\mathrm{K}$ oral anticoagulants a reasonable alternative to warfarin? J Am Heart Assoc. 2019;8:e012102.

13. Blann AD, Lip GYH. Laboratory monitoring of the non-vitamin K oral anticoagulants. J Am Coll Cardiol. 2014;64:1140-2.

14. Eikelboom JW, Connolly SJ, Brueckmann M, Granger CB, Kappetein AP, Mack MJ, et al. Dabigatran versus warfarin in patients with mechanical heart valves. $N$ Engl J Med. 2013;369:1206-14.

15. PROACT Xa-a trial to determine if participants with an On-X aortic valve can be maintained safely on apixaban. Available at: https://clinicaltrials.gov/ct2/ show/NCT04142658. Accessed January 18, 2021.
16. Sousa-Uva M, Head SJ, Milojevic M, Collet J-P, Landoni G, Castella M, et al. 2017 EACTS guidelines on perioperative medication in adult cardiac surgery. Eur J Cardiothorac Surg. 2018;53:5-33.

17. Erdoes G, Martinez Lopez de Arroyabe B, Bolliger D, Ahmed AB, Koster A, Agarwal S, et al. International consensus statement on the peri-operative management of direct oral anticoagulants in cardiac surgery. Anaesthesia. 2018;73: $1535-45$.

18. Heidbuchel H, Verhamme P, Alings M, Antz M, Diener H-C, Hacke W, et al. Updated European Heart Rhythm Association practical guide on the use of non-vitamin $\mathrm{K}$ antagonist anticoagulants in patients with non-valvular atrial fibrillation. Europace. 2015;17:1467-507.

19. Bayer-Westendorf J, Gelbricht V, Forster K, Ebertz F, Kohler C, Werth S, et al. Peri-interventional management of novel oral anticoagulants in daily care: results from the prospective Dresden NOAC registry. Eur Heart J. 2014;35:1888-96.

20. Hassan K, Bayer N, Schlingloff F, Oberhoffer M, Wohlmuth P, Schmoeckel M, et al. Bleeding complications after use of novel oral anticoagulants in patients undergoing cardiac surgery. Ann Thorac Surg. 2018;105:702-8.

21. Vranckx P, Potpara T, Dagres N, Heidbuchel H. Non-vitamin K oral anticoagulants in patients with atrial fibrillation after cardiac surgery: the results of the European Heart Rhythm Association Survey. Europace. 2016;18:1113-6.

22. Beller JP, Krebs ED, Hawkins RB, Mehaffey JH, Quader MA, Speir AM, et al. Non-vitamin K oral anticoagulant use after cardiac surgery is rapidly increasing. J Thorac Cardiovasc Surg. 2020;160:1222-31.

23. Seeger J, Gonska B, Rodewald C, Rottbauer W, Wohrle J. Apixaban in patients with atrial fibrillation after transfemoral aortic valve replacement. J Am Coll Cardiol Intv. 2017;10:66-74.

24. Dangas GD, Tijssen JGP, Wohrle J, Sondergaard L, Gilard M, Mollmann H, et al. A controlled trial of rivaroxaban after transcatheter aortic valve replacement. $N$ Engl J Med. 2020;382:120-9.

25. Woldendorp K, Khadra S, Bannon PG, Robinson BM. Novel oral anticoagulants compared with warfarin for postoperative atrial fibrillation after isolated coronary artery bypass grafting. Heart Lung Circ. 2020;29:1832-8.

26. Yu P-J, Lin D, Catalano M, Cassiere H, Manetta F, Kohn N, et al. Impact of novel oral anticoagulants vs warfarin on effusions after coronary artery bypass grafting. J Card Surg. 2019;34:419-23.

27. Anderson E, Johnke K, Leedahl D, Glogoza M, Newman R, Dyke C. Novel oral anticoagulants vs warfarin for the management of postoperative atrial fibrillation: clinical outcomes and cost analysis. Am J Surg. 2015;210:1095-103.

28. Chapin TW, Leedahl DD, Brown AB, Pasek AM, Sand MG, Loy ML, et al. Comparison of anticoagulants for postoperative atrial fibrillation after coronary artery bypass grafting: a pilot study. J Cardiovasc Pharmacol Ther. 2020;25:523-30.

29. Nauffal V, Trinquart L, Osho A, Sundt TM, Lubitz SA, Ellinor PT. Non-vitamin $\mathrm{K}$ oral anticoagulant vs warfarin for post cardiac surgery atrial fibrillation. Ann Thorac Surg. January 10, 2021 [Epub ahead of print].

30. Crowther M, Cuker A. How can we reverse bleeding in patients on direct oral anticoagulants? Kardiol Pol. 2019;77:3-11.

31. Carpenter E, Singh D, Dietrich E, Gums J. Andexanet alfa for reversal of factor Xa inhibitor-associated anticoagulation. Ther Adv Drug Saf. 2019;10. 2042098619888133

32. Pollack CV Jr, Reilly PA, van Ryn J, Eikelboom JW, Glund S, Bernstein RA, et al. Idarucizumab for dabigatran reversal—full cohort analysis. N Engl J Med. 2017; $377: 431-41$.

Key Words: nonvitamin $\mathrm{K}$ oral anticoagulant, anticoagulation 